\title{
Small Extracellular Vesicles Released from Glioma Cells Under Hypoxic Conditions Induce Pericyte- phenotype Transition of Glioma Stem Cells.
}

\section{Yue Cheng}

chong qing yi ke da xue: Chongqing Medical University

\section{Shijie Li}

Chongqing Medical University

\section{Yongying Hou}

Chongqing Medical University

\section{Weijun Wang}

Chongqing Medical University

\section{Ke Wang}

Chongqing Medical University

Ye Yuan ( $\nabla$ yuanye02@tmmu.edu.cn )

Army Medical University https://orcid.org/0000-0001-9892-2940

\section{Xiufeng Ye}

Chongqing Medical University

Primary research

Keywords: GBM, sEVs, GSC, TGF- $\beta 1$, Pericyte-phenotype Transition

Posted Date: April 12th, 2021

DOI: https://doi.org/10.21203/rs.3.rs-291935/v2

License: (9) This work is licensed under a Creative Commons Attribution 4.0 International License.

Read Full License 


\section{Abstract}

Background:The prognosis of malignant glioblastoma (GBM) is dismal despite advances in surgery, radiation and chemotherapy treatments. Thus, alternative therapy strategies are urgently needed. Antiangiogenic therapy for cancer offers the possibility of universal efficacy. However, preclinical and clinical studies suggest that this therapy using anti-VEGF drug Avastin (Bevacizumab) may lead to a promigratory phenotype in therapy resistant GBM and thus actively promote tumor invasion and recurrent tumor growth.

Methods: An ultracentrifugation strategy was used to isolate glioma-derived sEVs under hypoxic or normoxic conditions. Transmission electron microscopy (TEM), Western blotting, and nanoparticle tracking analysis (NTA) were used to characterize these isolated particles. Cytochalasin D was added to disrupt cellular sEVs uptake. A tube formation assay was used to evaluate angiogenic activity, while ELISAs and Western blotting were used to assess the activated TGF- $\beta$ signaling pathway. The effects of

sEVs on glioma stem cells (GSCs) in vivo were evaluated using subcutaneous xenografts model system in nude mice. Immunofluorescence and immunohistochemical staining were set out to evaluate the pericyte-phenotype transition of GSCs.

Results: In this present study, we showed that hypoxia could promote the release of sEVs by glioblastoma cells and hypoxia-induced glioma-derived sEVs could be taken up by GSCs. This internalization of sEVs promoted tumor growth in mouse Xenografts through the pericyte-phenotype transition of GSCs. We also demonstrated hypoxia-derived sEVs can efficiently deliver TGF- $\beta 1$ to GSCs. The activated TGF- $\beta$ signaling pathway mediated this kind of phenotype transition. In addition, combination of Ibrutinib and Bevacizumab showed more effective in targeting GBM.

Conclusion: This present study provides a new interpretation to the failure of antiangiogenesis therapy in noncurative surgical resection of GBM, and discovers promising brain-specific therapeutic targets for this damaging tumor.

\section{Introduction}

Glioblastoma (GBM) is the most common primary malignant brain tumors, with an annual incidence of about 5 per 100000 population and median survival 14.6 to 16 months with estimated 5 years survival rate of only $3.4 \%(1,2)$. Standard first line management for newly diagnosed GBM involves a multimodality approach, including surgical resection, followed by radiation with concurrent and adjuvant temozolomide(3). Even with optimal surgical resection and chemo-radiation, however, essentially all excised tumors will recur, and after recurrence, the two year survival is less than $30 \%(4,5)$. Thus, alternative therapy strategies are urgently needed. One of the most distinct characteristics of GBM is the aggressive angiogenesis (6). Thus, it seems promising to perform antiangiogenic therapy to treat GBM.

As proposed by Judah Folkman in 1971, prevention of new vessel sprouts or "antiangiogenesis" could result in tumor dormancy and thus be a novel cancer therapy $(7,8)$. Antiangiogenic therapy for cancer 
offers the possibility of universal efficacy, low toxicity, and little possibility of resistance(9). Blockade of the vascular endothelial growth factor (VEGF) pathway has yielded the most promising results both in animal models and in patients $(10,11)$. Bevacizumab (Avastin), an anti-VEGF monoclonal antibody, was grant FDA approval for use in treating recurrent GBM, with high initial radiographic response and disease control rates but most patients will progress after only a few months $(12,13)$. Upon recurrence, tumors may not enhance, secondary to vascular normalization. In addition, other recent prospective comparisons of Bevacizumab with other chemotherapeutic regimens have shown no significant increase in overall survival (OS) $(14,15)$. The mechanisms of Bevacizumab failure are likely complex and multifactorial. In this study, we emphasized the importance of GSCs in playing a vital role in the failure of antiangiogenic therapy.

GSCs promote GBM pathogenesis and contribute to the suboptimal efficacy of current treatment options through mediating angiogenesis, resistance to radiotherapy and chemotherapy, invasion into normal brain, and self-renewal (16). GSCs typically reside in specific intratumoral niches, such as the hypoxic core of a tumor or the perivascular niche (17). Once the blood vessels were disrupted by antiangiogenic therapy in tumor, GSCs then exposed to an artificial hypoxic microenvironment. There was an excitement generated by reports suggesting that GSCs may trans-differentiate into pericytes(18). This kind of phenotypic transformation promotes angiogenesis, helps repair the damaged endothelial cells and accelerate tumor growth(19). Within this context, we hypothesized that this kind of artificial hypoxic microenvironment caused by antiangiogenic therapy could induce GSCs to trans-differentiate into pericytes to sustain vascular normalization, making "antiangiogenesis" failure even distant or diffuse recurrence. In addition, this kind of phenotypic transition was endowed by small extracellular vesicles (sEVs),which were secreted by surrounding glioma cells under hypoxic condition.

SEVs have been described to underlie GBM initiation, invasion, angiogenesis, modulation of the immune response and chemoradiotherapy resistance(20). These particles ( $<200 \mathrm{~nm}$ diameter) are produced when polycystic intracellular structures fuse with the cell membrane(21). SEVs secreted to extracellular microenvironment are involved in the cell-cell neighboring communication and distant cells, favoring proteins, DNA, and mRNAs/miRNAs from donor cells to recipient cells(22). The secretory quantity of sEVs and their contents vary according to their biogenesis, cell of origin and cell's status. Accumulating evidence has demonstrated that the biogenesis of secretory growth factors in SEVs are tightly controlled, and dysregulation of these factors is linked to various cancers' initiation and progression $(23,24)$. Furthermore, sEV delivered growth factors interact with Cancer stem cells (CSCs) to confer tumoradvantageous changes that influence tumor progression and drug resistance $(25,26)$.

However, the knowledge regarding the interaction between GBM-derived SEVs and GSCs remains unknown. Whether this secretion of $\mathrm{sEVs}$ is induced by hypoxic microenvironment is unclear. Exploring the mechanisms behind this will help us not only to understand the biology of interaction between GBM cells and GSCs, which is thought to explain the failure of antiangiogenic therapy in noncurative surgical resection of gliomas, but also to discover promising brain-specific therapeutic targets for this damaging tumor. 
In this study, our findings revealed that sEVs derived from hypoxic GBM cells induce the pericytephenotype transition of GSCs through TGF- $\beta$ pathway signaling, which attenuates the therapeutic effect of "antiangiogenesis". Combination of Ibrutinib and Bevacizumab showed a stronger effectiveness in targeting GBM.

\section{Methods}

\section{Cell Culture}

U87, U251, and HUVEC cells were cultured in Dulbecco's Modified Eagle Medium (DMEM, GIBICO, C11995500BT) containing 10\% FBS (GIBCO, a31608-02) and 1\% penicillin/streptomycin (Beyotime, C0222). GSCs were derived from U87 and U251 cells using serum-free DMEM/F12 containing $20 \mathrm{ng} / \mathrm{mL}$ basic fibroblast growth factor (bFGF; Peprotech, 100-18b), $20 \mu \mathrm{L} / \mathrm{mL}$ B27 supplement (GIBC0,17504044), and $20 \mathrm{ng} / \mathrm{mL}$ epidermal growth factor (EGF; Peprotech,AF-100-15). GSC-like neurospheres grew under these conditions after 3 days and were digested using papain $(G I B C 0,88280)$ before 1:2 passage. Cells were routinely cultured in a humidified $37^{\circ} \mathrm{C} 5 \% \mathrm{CO}_{2} 95 \%$ air incubator. Hypoxic cell culture was conducted in an incubator containing $1 \% \mathrm{O}_{2}, 94 \% \mathrm{~N}_{2}$, and $5 \% \mathrm{CO}_{2}$ in an oxygen-regulated incubator .

\section{GSC Differentiation Analyses}

U87 cell-derived GSC-like cells were randomly separated into five treatment groups, after which growth media was replaced with DMEM containing $10 \%$ FBS after $0,2,4,6$, or 8 days. Cellular proteins were then isolated following $0,2,4,6$, or 8 days of growth, and CD133, SOX2, GFAP, and Nestin expression levels were assessed via Western blotting.

\section{SEV Isolatio $n$}

Cells were grown until $70 \%$ confluent, at which time media was exchanged for DMEM containing $10 \%$ sEV-free FBS (prepared via ultracentrifugation at $120,000 \times \mathrm{g}, 4^{\circ} \mathrm{C}$ for $18 \mathrm{~h}$ ) and $1 \%$ penicillin/streptomycin. Cells were then cultured for $48 \mathrm{~h}$ under normoxic $\left(37^{\circ} \mathrm{C} 5 \% \mathrm{CO}_{2} 95 \%\right.$ air $)$ or hypoxic $\left(1 \% \mathrm{O}_{2}, 94 \% \mathrm{~N}_{2}\right.$, and $5 \% \mathrm{CO}_{2}$ ) conditions as mentioned above, after which the media was collected and $\mathrm{sEV}$ were isolated via differential centrifugation. Briefly, cellular debris was removed by spinning samples for 10 minutes at 300 $\times \mathrm{g}$. Media was then spun for 30 minutes at $10,000 \times \mathrm{g}$ at $4^{\circ} \mathrm{C}$, following by passage through a $0.22 \mu \mathrm{m}$ filter. Filtering media were then isolated via ultracentrifugation for 90 minutes at $120,000 \times \mathrm{g}$ (Beckman Coulter, Optima XPN-100, USA) at $4^{\circ} \mathrm{C}$. Precipitated sEV were resuspended in PBS and subjected to a second round of ultracentrifugation under identical conditions, after which they were resuspended in $300 \mathrm{uL}$ PBS at $4^{\circ} \mathrm{C}$ and used for downstream analyses. sEV was stored at $-80^{\circ} \mathrm{C}$ for long term.

\section{Limiting Dilution Assay}

Limiting dilution assay was performed as previously described (27). In breif, GSC-like spheroid cells were cultured for a minimum of 7 days in serum-free media, then plated in $96-$-well plates $(200 \mathrm{uL} /$ well serumfree media), being diluted to concentrations of 20-240 cells/well. Every three days for 14 days, an 
additional $25 \mathrm{uL}$ of serum-free media was added to each well. Spheres larger than 50 um were then counted, and a regression approach was used to determine the minimum number of cells necessary to give rise to a minimum of 1 tumorsphere per well.

\section{Assessment of SEV Internalization by GSCs and HUVEC Cells}

The fluorescent PKH26dyes (MINI26-1KT, Sigma-Aldrich, USA) or PKH67dyes (green, Sigma-Aldrich, USA) were used to label sEV by combining $100 \mu \mathrm{g}$ of sEV in $200 \mathrm{uL}$ of PBS with $1 \mathrm{~mL}$ of an appropriately provided diluent and $1 \mathrm{uL}$ of $\mathrm{PKH} 67$ or $\mathrm{PKH} 26$ for 3-5 minutes. A $1 \mathrm{~mL}$ volume of FBS was then added to terminate labeling, and SEV were washed with PBS and reisolated via ultracentrifugation. Staining was conducted at room temperature. In order to assess sEV internalization, GSCs or HUVECs were cultured for $12 \mathrm{~h}$ in DMEM containing $10 \% \mathrm{sEV}$-free FBS on glass covers lips. The media was exchanged for media containing labeled sEV $(20 \mu \mathrm{g} / \mathrm{ml})$, and cells were cultured for an additional $12 \mathrm{~h}$ at $37^{\circ} \mathrm{C}$. Cells were then fixed using 4\% paraformaldehyde (PFA) and stained overnight with a-tubulin (1:200, CST, 2144, USA). Cells were then washed with PBS, stained using DAPI, blocked with an anti-fluorescence quencher, and imaged via fluorescence microscope(Leica, DMi8, Germany).

\section{Western Blotting}

Western blotting was performed as previously described(28). SEV and cells were lysed using RIPA buffer supplemented with $1 \%$ PMSF. Proteins were separated via SDS-PAGE and transferred to PVDF membranes. Blots were then probed using antibodies specific for CD63 (Proteintech, 25682-1-AP, USA), TSG101 (Abcam, ab125011, USA), CD9 (Abcam, ab92726, USA), CD81 (Abcam, ab79559, USA), GRP94 (Abcam, ab238126, USA), Alix (Proteintech, 12422-1-AP, USA), B-actin (CST, 3700, USA), GAPDH(CST, 5174, USA), Nestin (Proteintech, 19483-1-AP, USA), Sox2 (Proteintech, 11064-1-AP, USA), GFAP (Proteintech, 16825-1-AP, USA), TGF- 31 (Proteintech, 21898-1-AP, USA), Smad2 (CST, 5339, USA), Smad3 (CST, 9523, USA), p-Smad2 (CST, 18338, USA), p-Smad3 (CST, 8828, USA), BMX (CST, 8828, USA), CD133 (CST, 64326, USA), a-SMA (CST, 19245, USA), PDGFRß(CST, 3169, USA), CD146 (Proteintech, 66153-1-Ig, USA), and HIF-1a (CST, 36169, USA). Secondary antibodies (Servicebio) and ECL (Advasta, K-12045-D10, USA) were then utilized for protein band detection.

\section{Migration Assays}

GSCs $\left(2 \times 10^{4}\right)$ were added to the upper chamber of a Transwell insert (BD, 353097, USA) in a 24-well plate in serum-free DMEM/F12. DMEM containing 10\% sEV-free FBS was then added to the lower chamber, and cells were incubated for $12,24,48$, or $72 \mathrm{~h}$. Cotton swabs were then used to carefully remove non-migratory cells, while cells that had migrated were fixed for 30 minutes with $4 \%$ PFA, washed with PBS, stained using crystal violet, and counted via microscopy. Three random fields of view in triplicate samples were analyzed for this assay.

\section{qRT-PCR}


Trizol (Invitrogen, 15596026, USA) was used to isolate total RNA from GSCs, after which RNase-free DNase I (Biosharp, BL510A, China) was used to treat this RNA. A BcaBest RNA PCR kit (TaKaRa, RR023B, Japan) was then used to prepare cDNA based on provided directions, and qRT-PCR was conducted with an iQ5 Multicolor Real-Time PCR Detection System (Bio-Rad) using a Realtime PCR Master Mix (SYBR Green, RR064B, Japan) and the primers listed below, with actin being used as an endogenous control:

ß-actin, forward: 5-AGATGTGGATCAGCAAGCA-3,

reverse, 5-GCGCAAGTTAGGTTTTGTCA-3;

CD146, forward: 5- GTCAATTTAACCACCCTCACAC-3,

reverse: 5-GCTTGCCCTTCTTATAGAGGAA-3;

PDGFR, forward: 5-TGTGAATGACCATCAGGATGAA-3,

reverse: 5-CAGCTCAGCAAATTGTAGTGTG-3;

a-SMA, forward: 5-GAGGACCTCTGTTCTGTGCATTGG-3,

reverse: 5-GCGGCTGCCTAGTGTACTTGC-3.

Desmin, forward: 5-CCCAACAGCGACATAGCCCATC-3,

reverse, 5-CACTCACTGCCAACAGCCAAGG-3;

Sox2, forward: 5-ACATGAACGGCTGGAGCAACG-3,

reverse, 5-CTGCGAGCTGGTCATGGAGTTG-3;

CD133, forward: 5-TCGCCTCAAGCCAGCCTCAG-3,

reverse, 5-ACTTGGTGCCTCCTGCCTCAG-3.

\section{Immunofluorescence and Hematoxylin and Eosin (H\&E) Staining}

Cells or spheroids on poly-l-lysine-coated (Sigma, P4707, USA) glass coverslips were fixed for 30 minutes using 4\% PFA, then washed three times in PBS. Tumor tissue samples were also fixed with 4\% PFA prior to paraffin embedding and cutting into 6 um-thick sections. Samples were blocked for 30 minutes using goat serum, followed by overnight incubation with appropriate primary antibodies in $5 \%$ BSA at $4{ }^{\circ} \mathrm{C}$. The following antibodies were used for this analysis: GFAP (1: 200, Proteintech, 16825-1-AP, USA), SOX2 (1:200, Proteintech, 11064-1-AP, USA), CD133 (1:50, CST, 64326, USA) and Nestin (1:100, Proteintech, 19483-1-AP, USA). Cells were then washed and stained using secondary fluorescently-labeled antibodies (1:200, Proteintech, 15031 and SA00009-2, USA) for $1 \mathrm{~h}$ at room temperature. Samples were then washed 
with PBS and stained using DAPI. For H\&E staining, tissue sections were stained using standard H\&E staining methods. Optical or confocal microscopes (Leica, DMi8, Germany) were used to image these samples.

\section{ELISAs}

Levels of TGF- $\beta 1$ in cells, sEV, or culture supernatants were assessed using ELISA kits (Elabscience, E-EL0162c, China) based on provided directions.

\section{Tube Formation Assay}

HUVECs and GSCs $\left(3 \times 10^{4}\right)$ were grown in Matrigel-coated 96-well plates (BD, 356234, USA) for $6 \mathrm{~h}$ at $37^{\circ} \mathrm{C}$. Cells were then cultured with hypoxic or normoxic sEV $(50 \mathrm{ug} / \mathrm{ml})$, with PBS added as a control and cytochalasin D $(5 \mathrm{ug} / \mathrm{ml})$ added to appropriate wells as above. Capillary-like tube formation was assessed over time via light microscopy, and Image J was used to quantify the degree of angiogenesis based upon the number of branch points formed in these tubes.

\section{Xenografts Model}

The Chongqing Medical University Animal Care and Use Committee approved all animal studies, which were consistent with the guidelines of the National Institutes of Health. In GSC and U87 experiments, female BALB/c mice (4-6-week-old, 18-20 g) were subcutaneously implanted with $1 \times 10^{6}$ tumor cells in a $200 \mu \mathrm{L}$ volume in the back thigh. U87 and GSCs were implanted on the left and right flanks, respectively. In GSC and sEV experiments, female BALB/c mice (4-6-week-old, 16-18 g) were implanted with tumor cells in the right flank as mentioned above, and were then randomly separated into six treatment groups, with SEV administration being initiated on day 7 post-implantation. Drug treatments were initiated on day 21 post-implantation. Treatment groups were as follows: PBS, normoxic sEV 100ug in 100ul PBS 3 times a week), hypoxic sEV (100 ug in 100ul PBS 3 times a week), hypoxic sEV + Ibrutinib (Aladdin, P127143-10MG, China) (inhibitor group 1, $15 \mathrm{mg} / \mathrm{kg}$ every day), hypoxic sEV + Bevacizumab (Avastin, Switzerland) (inhibitor group 2, 25mg/kg every 2 days), or hypoxic sEV + Ibrutinib + Bevacizumab (inhibitor group 3). Tumor volume and body weight were assessed every 3 days; tumor volume (V) was determined by measuring tumor length (a) and width (b), using the formula $V=(a b 2) / 2$.

\section{Statistical Analysis}

Image $\mathrm{J}$ was used to analyze the gray values of Western blot bands and to count the cells of Transwell results. Statistical analysis was performed using GraphPad Prism 6.0. Data were presented as ' $x \pm s$, and differences between the two groups were analyzed by independent sample $t$ test. Data were deemed to be significantly different at $P$ values less than 0.05 .

\section{Result}

The Internalization of Hypoxia-Induced Glioma-Derived SEVs by GSCs Promote Tumor Growth in a Mouse Xenograft. 
Hypoxia is an important contributor to aggressive behavior and resistance mechanisms in GBM. We asked whether hypoxia could promote the release of sEVs by GBMs and hypoxia-induced glioma-derived sEVs could be taken up by GSCs. We first isolated sEVs from the culture supernatants of human glioma cell lines U87 and U251 in the absence of oxygen $\left(1 \% \mathrm{O}_{2}\right)$ by density gradient centrifugation. SEVs from the two cell lines exhibited a double concave tea-dish-like morphology as determined by TEM, and hypoxia did not affect this morphology (Fig. 1A). In addition, particle size and concentration analysis by NTA approach demonstrated that hypoxia-induced sEVs diameter is $110 \mathrm{~nm}$, and hypoxia did not change the sEV particle diameter distribution (Fig. 1B). We further validated the presence of the known sEV markers: CD63, TSG101, CD81, CD9 and the absence of the negative markers: GRP94, GAPDH, $\beta$ actin, and a-tubulin by Western blot assay (Fig. 1C). Then, we sorted and identified GSCs from U87 and U251 cell lines as previously reported (Supplementary Fig. 1). SEVs were stained with the PKH67(Green) lipophilic dyesin advance. Confocal microscopy was used to measure the internalization of sEVs by GSCs after 24h incubation, and microscopy analysis of the GSCs demonstrated an increasing uptake of labeled sEVs (Fig. 1D). We asked whether this internalization could promote proliferation of GBM cells. Thus, we created U87-GSCs Xenografts model using nude mice, and intraperitoneally injected them with PBS or normoxia/hypoxia-derived sEVs. After 2-week treatment, tumor volumes showed significant differences between each groups (Fig. 1E). Taken together, these results show the internalization of hypoxia-induced glioma-derived sEVs by GSCs promotes tumor growth in a mouse Xenograft.

\section{SEVs Derived from Hypoxic Glioma Cells Induce Pericyte-Phenotype Transition of GSCs.}

To investigate the exact effects of hypoxia-derived sEVs on promoting tumor growth. We asked whether the hypoxia-induced sEVs could induce pericyte transition of GSCs. Notably, immunohistochemical and fluorescence staining of endothelial and pericyte markers, such as CD31 and a-SMA, were significantly increased after treated with hypoxic sEVs, indicating the increased pericyte differentiation and angiogenesis in vivo. To our surprise, we also found that hypoxia-derived sEVs increased the expression of perivascular bone marrow and X-linked (BMX), which is preferentially up-regulated in GSCs(29). This indicates that GSCs migrates to the perivascular niche prior to adopting a pericyte-like phenotype (Fig. 2A, B). We then set out to evaluate the effects of hypoxia-derived sEVs on pericyte transition of GSCs in vitro. Notably, the pericyte markers, including PDGFR, CD146, Desmin, and a-SMA were significantly increased in hypoxia-derived sEVs treated U87 /U251 derived GSCs compared with these treated with normoxiaderived sEVs. These results were consistent with the decrease of GSC markers, such as CD133 and SOX2 (Fig. 2C, D). In addition, the enhanced tube formation abilities, including increased branching, branch length, junction numbers, total branching length, and the total length of these tubules, were observed in GSCs treated with hypoxia-derived sEVs compared with these treated with normoxia-derived sEVs or PBS. In addition, these enhanced tube formation activities can be attenuated by Cyt D ((Fig. 2E, F). These results suggest that hypoxia-derived sEVs induces the transition of GSCs to pericyte phenotype.

\section{TGF- $\beta$ Signaling Pathway Mediates the Pericyte-Phenotype Transition of GSCs.}


Next, we aimed to understand the underlying mechanism why hypoxia-derived sEVs can induce pericytephenotype transition of GSCs. As has been mentioned in a previous study, GSCs generate vascular pericytes to support vessel function and tumor growth predominantly by TGF- $\beta(30)$. We firstly downloaded a microarray (GSE101113), which contains mRNA data of 56 polyclonal and monoclonal glioma stem cell lines, from GEO Database. Gene Set Enrichment Analysis (GSEA) was performed with KEGG pathway analysis, the results showed that the stemness marker of GSCs, CD15 (encoded by FUT4), was positively correlated with TGF- $\beta$ signaling pathway (Fig. 3A). Thus, we determined whether TGF- $\beta$ signaling pathway mediates this kind of phenotype transition. Notably, when we added hypoxia-derived sEVs to U87/U251 derived GSCs, the protein level of TGF- $\beta 1$, p-Smad2, and p-Smad3 were upregulated, while the expression of Smad2 and Smad3 remained unchanged, indicating the activation of TGF- $\beta$ signaling pathway (Fig. 3B). We then questioned whether the upregulated TGF- $\beta 1$ in GSCs was delivered by hypoxia-derived sEVs. Therefore, we utilized ELISA to evaluate TGF- $\beta 1$ expression in sEVs. We observed that TGF- $\beta 1$ were upregulated to a greater level under hypoxic conditions than normoxic conditions (Fig. 3C). The upregulated TGF- $\beta 1$ was also observed in hypoxia-derived sEVs, which were tested by western blotting (Fig. 3D). In addition, we set out to conduct rescue experiment using anti-TGF$\beta 1$. Notably, the pericyte markers, including PDGFR, CD146, Desmin, and a-SMA, were significantly decreased in U87/U251 derived GSCs treated with anti-TGF- $\beta 1$. Taken together, these results suggest that TGF- $\beta 1$ signaling pathway mediates the pericyte-phenotype transition of GSCs.

\section{Combination of Ibrutinib and Bevacizumab Significantly Inhibits Tumor Growth In Vivo.}

Since we have found that hypoxic microenvironment, which can be caused by Bevacizumab treatment in clinical practice, could induce the pericyte-phenotype transition of GSCs through hypoxia-derived sEVs. We then asked whether combination of Ibrutinib and Bevacizumab could enhance the therapeutic effect of "antiangiogenesis". Therefore, we established U87-GSCs Xenografts model and treated them with $1 \%$ $02 \mathrm{sEVs}$. We observed that the tumor size was significantly reduced by the application of Ibrutinib, or Bevacizumab, or together. Whatsmore, combination of Ibrutinib and Bevacizumab could mostly reduce the tumor growth compared with each alone (Fig. 4A). Notably, we observed that the staining of a-SMA and CD31 were significantly downregulated, consistent with the decrease of BMX, indicating angiogenesis and pericyte-phenotype transition of GSCs. These results were in accord with the group using the combination treatment of Ibrutinib and Bevacizumab. In addition, we also observed that Ki67 was significantly decreased. This indicates the use of Ibrutinib and Bevacizumab could not only target GSCs and endothelial cells but also glioma cells.( Fig. 4B, C) Taken together, these findings demonstrate combination of Ibrutinib and Bevacizumab can significantly inhibit tumor growth in vivo.

\section{Discussion}

The prognosis of malignant gliomas is still dismal despite advances in surgery, radiation and chemotherapy treatments, the overall 5 -year survival rate for people with this type of tumor remains $<10 \%$ (31). Thus, alternative therapy strategies are needed. Malignant gliomas are upon the best vascularized tumors in humans and their proliferation is hallmarked by a distinct proliferative vascular 
component(32). Hence it seems to be a logical consequence to apply anti-angiogenic treatment strategies to malignant gliomas. These treatment strategies have shown promising effects in animal models and some experimental clinical studies. The antiangiogenesis drug Avastin (Bevacizumab), which targets VEGF, has become one of the most popular cancer drugs in the world(33). Anti-angiogenic therapy may lead to vascular normalization and as such facilitate conventional cytotoxic chemotherapy. However, preclinical and clinical studies suggest that anti-VEGF therapy using bevacizumab may also lead to a pro-migratory phenotype in therapy resistant GBMs and thus actively promote tumor invasion and recurrent tumor growth(34). The mechanisms of Bevacizumab failure are likely complex and multifactorial. Over time, tumors demonstrate adaptive upregulation of alternative angiogenic pathways. One explaination is that hypoxic microenvironment caused by the treatment of Bevacizumab accelerate the formation of abnormal vasculature. However, knowledge regarding this is limited.

In this study, we found that hypoxia could promote the release of sEVs by GBMs and hypoxia-induced glioma-derived sEVs could be taken up by GSCs. In addition, this internalization of hypoxia-derived sEVs promoted tumor growth in a mouse Xenograft through the pericyte-phenotype transition of GSCs. TGF- $\beta 1$ signaling pathway mediates this phenotype transition. It is well known that sEVs constitute an outstanding communication system in GBM, they can deliver not only a wide variety of coding and noncoding RNAs, but soluble proteins that can alter gene expression and cellular function in recipient cells $(35,36)$. Evidence from the last few years show that GBMs interact with surrounding non-cancer cells through SEVs to maintain a microenvironment that favors tumor proliferation, invasion of the brain, angiogenesis and immunosuppression(37). Franz $L$ et al. reported the immune evasion mediated by PDL1 on GBM-derived sEVs(38). In addition, sEVs are recognized as a important component in modifying cellular phenotype. For example, Oushy et al. showed GBM-derived sEVs drive normal astrocytes towards a tumour-enhancing phenotype(39). Jeroen et al. reported GBM-derived SEVs can modify cells of the monocytic lineage to acquire characteristics that resemble the tumor-supportive phenotypes(40). Although the communication between sEVs released by GBM and non-cancer cells, including monocytes, macrophages, microglia, endothelial cells, astrocytes, and mesenchymal stem cells, has been fully investigated(41-45). The knowledge regarding the interaction between GBM-derived sEVs and GSCs is limited. As we all known, the surrounding GBM cells are the closest and most important microenvironment of GSCs in term of quantity and distance. Thus, there is a need to further understand the interaction between GBMs and GSCs through not just the reported direct or indirect communication, including cell junction, paracellular secretory and endocrine pathway(46-48). Based on our study, GSCs could internalize hypoxia-derived SEVs to accomplish pericyte-phenotype transition. It has been reported that high pericyte coverage of GBM blood vessels is associated with poor response to chemotherapy in human patients(49). Thus, this kind of phenotypic transformation can attenuate the Inhibition of Bevacizumab ,which prunes abnormal blood vessels and "normalizes" vasculature into thin-walled vessels composed of a single layer of endothelial cells in GBM.

Based on these findings, we expect that inhibition of VEGF combined with GSCs can further improve the efficacy in treating GBM. It has been previously reported that BMX is an essential factor for maintaining GSCs-derived pericytes and inhibiting BMX with ibrutinib selectively targeted neoplastic pericytes and 
disrupts the blood-tumor barrier (BTB) (50). In this present study, Ibrutinib combined with Bevacizumab was utilized to treat U87-GSCs Xenografts, which were previously injected with hypoxia-derived sEVs. Notably, this combination therapy could mostly reduce the tumor growth compared with each alone. On the other hand, in addition to normalizing vasculature, Bevacizumab is also thought to have direct antitumor activity against GBM cells that express VEGF(51). Our results show the staining of Ki67 in Xenograft was significantly decreased after the treatment of Bevacizumab alone. This is consistent with the previous study.

In summary, our findings revealed that sEVs derived from hypoxic GBM cells induce the pericytephenotype transition of GSCs through TGF- $\beta$ pathway signaling, thus attenuating the therapeutic effect of "antiangiogenesis". Combination of Ibrutinib and Bevacizumab showed more effective in targeting GBM. This present study gives a new interpretation to the failure of antiangiogenesis therapy, and could provide a promising therapeutic strategy in targeting GBM.

\section{Conclusion}

This present study provides a new interpretation to the failure of antiangiogenesis therapy in noncurative surgical resection of gliomas, and discover promising brain-specific therapeutic targets for this damaging tumor.

\section{Declarations}

\section{DATA AVAILABILITY STATEMENT}

The raw data supporting the conclusions of this article will be made available by the authors, without undue reservation.

\section{ETHICS STATEMENT}

The human tissues were obtained and studied in strict adherence to the protocol approved by the Institute Research Medical Ethics Committee of the Southwest Medical University. The animal study was reviewed and approved by Laboratory Animal Center of Chongqing, China.

\section{AUTHOR CONTRIBUTIONS}

$Y Y$ and $X Y$ spearheaded and supervised all of the experiments. $Y Y$ and $X Y$ designed this project. $Y C, S L$, $\mathrm{YH}, \mathrm{WW}$, and $\mathrm{KW}$ conducted the experiments. $\mathrm{YC}$ analyzed the data and provided the materials. $\mathrm{YY}$ and $Y C$ prepared the manuscript. $Y Y$ and $X Y$ revised the manuscript. All authors reviewed and approved the manuscript.

\section{FUNDINNG}

This work was supported by the Foundation for Science and Technology Research Project of Chongqing (CSTC2012ggB10002). 


\section{References}

1. Alexander BM, Cloughesy TF. Adult Glioblastoma. J CLIN ONCOL. 2017;35:2402-9. doi:10.1200/JC0.2017.73.0119.

2. Omuro A, DeAngelis LM. Glioblastoma and other malignant gliomas: a clinical review. JAMA. 2013;310:1842-50. doi:10.1001/jama.2013.280319.

3. Osuka S, Van Meir EG. Overcoming therapeutic resistance in glioblastoma: the way forward. J CLIN INVEST. 2017;127:415-26. doi:10.1172/JCI89587.

4. Bunevicius A, Sheehan JP. Radiosurgery for Glioblastoma. NEUROSURG CLIN N AM. 2021;32:11728. doi:10.1016/j.nec.2020.08.007.

5. Zhou Y, Wu W, Bi H, Yang D, Zhang C. Glioblastoma precision therapy: From the bench to the clinic. CANCER LETT. 2020;475:79-91. doi:10.1016/j.canlet.2020.01.027.

6. Broekman ML, Maas S, Abels ER, Mempel TR, Krichevsky AM, Breakefield XO. Multidimensional communication in the microenvirons of glioblastoma. NAT REV NEUROL. 2018;14:482-95. doi:10.1038/s41582-018-0025-8.

7. Bottsford-Miller JN, Coleman RL, Sood AK. Resistance and escape from antiangiogenesis therapy: clinical implications and future strategies. J CLIN ONCOL. 2012;30:4026-34. doi:10.1200/JC0.2012.41.9242.

8. Albini A, Tosetti F, Li VW, Noonan DM, Li WW. Cancer prevention by targeting angiogenesis. NAT REV CLIN ONCOL. 2012;9:498-509. doi:10.1038/nrclinonc.2012.120.

9. Gerstner ER, Batchelor TT. Antiangiogenic therapy for glioblastoma. CANCER J. 2012;18:45-50. doi:10.1097/PPO.0b013e3182431c6f.

10. Chen HX, Cleck JN. Adverse effects of anticancer agents that target the VEGF pathway. NAT REV CLIN ONCOL. 2009;6:465-77. doi:10.1038/nrclinonc.2009.94.

11. Loges $S$, Mazzone M, Hohensinner P, Carmeliet P. Silencing or fueling metastasis with VEGF inhibitors: antiangiogenesis revisited. CANCER CELL. 2009;15:167-70.

doi:10.1016/j.ccr.2009.02.007.

12. Mayer TM. Can We Predict Bevacizumab Responders in Patients With Glioblastoma? J CLIN ONCOL. 2015;33:2721-2. doi:10.1200/JC0.2015.62.3637.

13. Kim MM, Umemura Y, Leung D. Bevacizumab and Glioblastoma: Past, Present, and Future Directions. CANCER J. 2018;24:180-6. doi:10.1097/PPO.0000000000000326.

14. Diaz RJ, Ali S, Qadir MG, De La Fuente MI, Ivan ME, Komotar RJ. The role of bevacizumab in the treatment of glioblastoma. J Neurooncol. 2017;133:455-67. doi:10.1007/s11060-017-2477-x.

15. Funakoshi Y, Hata N, Kuga D, Hatae R, Sangatsuda Y, Fujioka Y, Takigawa K, Mizoguchi M. Update on Chemotherapeutic Approaches and Management of Bevacizumab Usage for Glioblastoma. Pharmaceuticals (Basel).(2020)13. doi:10.3390/ph13120470.

16. Pine AR, Cirigliano SM, Nicholson JG, Hu Y, Linkous A, Miyaguchi K, Edwards L, Singhania R, Schwartz TH, Ramakrishna R, Pisapia DJ, Snuderl M, Elemento O, Fine HA. Tumor Microenvironment 
Is Critical for the Maintenance of Cellular States Found in Primary Glioblastomas. CANCER DISCOV. 2020;10:964-79. doi:10.1158/2159-8290.CD-20-0057.

17. Woolard K, Fine HA. Glioma stem cells: better flat than round. CELL STEM CELL. 2009;4:466-7. doi:10.1016/j.stem.2009.05.013.

18. Cheng L, Huang Z, Zhou W, Wu Q, Donnola S, Liu JK, Fang X, Sloan AE, Mao Y, Lathia JD, Min W, McLendon RE, Rich JN, Bao S. Glioblastoma stem cells generate vascular pericytes to support vessel function and tumor growth. CELL. 2013;153:139-52. doi:10.1016/j.cell.2013.02.021.

19. Bernardes SS, Pinto M, Amorim JH, Azevedo V, Resende RR, Mintz A, Birbrair A. Glioma Pericytes Promote Angiogenesis by Producing Periostin. CELL MOL NEUROBIOL. 2020. doi:10.1007/s10571020-00975-3.

20. Lane R, Simon T, Vintu M, Solkin B, Koch B, Stewart N, Benstead-Hume G, Pearl F, Critchley G, Stebbing J, Giamas G. Cell-derived extracellular vesicles can be used as a biomarker reservoir for glioblastoma tumor subtyping. Commun Biol. 2019;2:315. doi:10.1038/s42003-019-0560-x.

21. Kosaka N, Yoshioka Y, Fujita Y, Ochiya T. Versatile roles of extracellular vesicles in cancer. J CLIN INVEST. 2016;126:1163-72. doi:10.1172/JCl81130.

22. Tkach M, Thery C. Communication by Extracellular Vesicles: Where We Are and Where We Need to Go. CELL. 2016;164:1226-32. doi:10.1016/j.cell.2016.01.043.

23. Xu R, Rai A, Chen M, Suwakulsiri W, Greening DW, Simpson RJ. Extracellular vesicles in cancer implications for future improvements in cancer care. NAT REV CLIN ONCOL. 2018;15:617-38. doi:10.1038/s41571-018-0036-9.

24. Huang T, Song C, Zheng L, Xia L, Li Y, Zhou Y. The roles of extracellular vesicles in gastric cancer development, microenvironment, anti-cancer drug resistance, and therapy. MOL CANCER. 2019;18:62. doi:10.1186/s12943-019-0967-5.

25. Lindoso RS, Collino F, Vieyra A. Extracellular vesicles as regulators of tumor fate: crosstalk among cancer stem cells, tumor cells and mesenchymal stem cells. Stem Cell Investig. 2017;4:75. doi:10.21037/sci.2017.08.08.

26. Nakano I, Garnier D, Minata M, Rak J. Extracellular vesicles in the biology of brain tumour stem cells-Implications for inter-cellular communication, therapy and biomarker development. SEMIN CELL DEV BIOL. 2015;40:17-26. doi:10.1016/j.semcdb.2015.02.011.

27. Yuan Y, Yan Z, Miao J, Cai R, Zhang M, Wang Y, Wang L, Dang W, Wang D, Xiang D, Wang Y, Zhang P, Cui Y, Bian X, Ma Q. Autofluorescence of NADH is a new biomarker for sorting and characterizing cancer stem cells in human glioma. STEM CELL RES THER. 2019;10:330. doi:10.1186/s13287-0191467-7.

28. Song K, Yuan Y, Lin Y, Wang YX, Zhou J, Gai QJ, Zhang L, Mao M, Yao XX, Qin Y, Lu HM, Zhang X, Cui $\mathrm{YH}$, Bian XW, Zhang X, Wang Y. ERBB3, IGF1R, and TGFBR2 expression correlate with PDGFR expression in glioblastoma and participate in PDGFR inhibitor resistance of glioblastoma cells. AM J CANCER RES. 2018;8:792-809. 
29. Shi Y, Guryanova OA, Zhou W, Liu C, Huang Z, Fang X, Wang X, Chen C, Wu Q, He Z, Wang W, Zhang W, Jiang T, Liu Q, Chen Y, Wang W, Wu J, Kim L, Gimple RC, Feng H, Kung HF, Yu JS, Rich JN, Ping YF, Bian XW, Bao S. Ibrutinib inactivates BMX-STAT3 in glioma stem cells to impair malignant growth and radioresistance. SCI TRANSL MED.(2018)10. doi:10.1126/scitranslmed.aah6816.

30. Cheng L, Huang Z, Zhou W, Wu Q, Donnola S, Liu JK, Fang X, Sloan AE, Mao Y, Lathia JD, Min W, McLendon RE, Rich JN, Bao S. Glioblastoma stem cells generate vascular pericytes to support vessel function and tumor growth. CELL. 2013;153:139-52. doi:10.1016/j.cell.2013.02.021.

31. Cappellano AM, Petrilli AS, Da SN, Silva FA, Paiva PM, Cavalheiro S, Bouffet E. Single agent vinorelbine in pediatric patients with progressive optic pathway glioma. J Neurooncol. 2015;121:405-12. doi:10.1007/s11060-014-1652-6.

32. Tuettenberg J, Friedel C, Vajkoczy P. Angiogenesis in malignant glioma-a target for antitumor therapy? Crit Rev Oncol Hematol. 2006;59:181-93. doi:10.1016/j.critrevonc.2006.01.004.

33. Wu HB, Yang S, Weng HY, Chen Q, Zhao XL, Fu WJ, Niu Q, Ping YF, Wang JM, Zhang X, Yao XH, Bian $X W$. Autophagy-induced KDR/VEGFR-2 activation promotes the formation of vasculogenic mimicry by glioma stem cells. AUTOPHAGY. 2017;13:1528-42. doi:10.1080/15548627.2017.1336277.

34. Plate KH, Scholz A, Dumont DJ. Tumor angiogenesis and anti-angiogenic therapy in malignant gliomas revisited. ACTA NEUROPATHOL. 2012;124:763-75. doi:10.1007/s00401-012-1066-5.

35. Lane R, Simon T, Vintu M, Solkin B, Koch B, Stewart N, Benstead-Hume G, Pearl F, Critchley G, Stebbing J, Giamas G. Cell-derived extracellular vesicles can be used as a biomarker reservoir for glioblastoma tumor subtyping. Commun Biol. 2019;2:315. doi:10.1038/s42003-019-0560-x.

36. Dobra G, Bukva M, Szabo Z, Bruszel B, Harmati M, Gyukity-Sebestyen E, Jenei A, Szucs M, Horvath P, Biro T, Klekner A, Buzas K. Small Extracellular Vesicles Isolated from Serum May Serve as SignalEnhancers for the Monitoring of CNS Tumors. INT J MOL SCl.(2020)21. doi:10.3390/ijms21155359.

37. Whitehead CA, Kaye AH, Drummond KJ, Widodo SS, Mantamadiotis T, Vella LJ, Stylli SS.

Extracellular vesicles and their role in glioblastoma. Crit Rev Clin Lab Sci.(2019)1-26. doi:10.1080/10408363.2019.1700208.

38. Ricklefs FL, Alayo Q, Krenzlin H, Mahmoud AB, Speranza MC, Nakashima H, Hayes JL, Lee K, Balaj L, Passaro C, Rooj AK, Krasemann S, Carter BS, Chen CC, Steed T, Treiber J, Rodig S, Yang K, Nakano I, Lee H, Weissleder R, Breakefield XO, Godlewski J, Westphal M, Lamszus K, Freeman GJ, Bronisz A, Lawler SE, Chiocca EA. Immune evasion mediated by PD-L1 on glioblastoma-derived extracellular vesicles. SCI ADV. 2018;4:eaar2766. doi:10.1126/sciadv.aar2766.

39. Oushy S, Hellwinkel JE, Wang M, Nguyen GJ, Gunaydin D, Harland TA, Anchordoquy TJ, Graner MW. Glioblastoma multiforme-derived extracellular vesicles drive normal astrocytes towards a tumourenhancing phenotype. Philos Trans R Soc Lond B Biol Sci.(2018)373. doi:10.1098/rstb.2016.0477.

40. de Vrij J, Maas SL, Kwappenberg KM, Schnoor R, Kleijn A, Dekker L, Luider TM, de Witte LD, Litjens M, van Strien ME, Hol EM, Kroonen J, Robe PA, Lamfers ML, Schilham MW, Broekman ML.

Glioblastoma-derived extracellular vesicles modify the phenotype of monocytic cells. INT J CANCER. 2015;137:1630-42. doi:10.1002/ijc.29521.

Page $14 / 20$ 
41. Bowman RL, Klemm F, Akkari L, Pyonteck SM, Sevenich L, Quail DF, Dhara S, Simpson K, Gardner EE, lacobuzio-Donahue CA, Brennan CW, Tabar V, Gutin PH, Joyce JA. Macrophage Ontogeny Underlies Differences in Tumor-Specific Education in Brain Malignancies. CELL REP. 2016;17:2445-59. doi:10.1016/j.celrep.2016.10.052.

42. Li Q, Barres BA. Microglia and macrophages in brain homeostasis and disease. NAT REV IMMUNOL. 2018;18:225-42. doi:10.1038/nri.2017.125.

43. Calabrese C, Poppleton H, Kocak M, Hogg TL, Fuller C, Hamner B, Oh EY, Gaber MW, Finklestein D, Allen M, Frank A, Bayazitov IT, Zakharenko SS, Gajjar A, Davidoff A, Gilbertson RJ. A perivascular niche for brain tumor stem cells. CANCER CELL. 2007;11:69-82. doi:10.1016/j.ccr.2006.11.020.

44. Brandao M, Simon T, Critchley G, Giamas G. Astrocytes, the rising stars of the glioblastoma microenvironment. GLIA. 2019;67:779-90. doi:10.1002/glia.23520.

45. Figueroa J, Phillips LM, Shahar T, Hossain A, Gumin J, Kim H, Bean AJ, Calin GA, Fueyo J, Walters ET, Kalluri R, Verhaak RG, Lang FF. Exosomes from Glioma-Associated Mesenchymal Stem Cells Increase the Tumorigenicity of Glioma Stem-like Cells via Transfer of miR-1587. CANCER RES. 2017;77:5808-19. doi:10.1158/0008-5472.CAN-16-2524.

46. Lathia JD, Mack SC, Mulkearns-Hubert EE, Valentim CL, Rich JN. Cancer stem cells in glioblastoma. Genes Dev. 2015;29:1203-17. doi:10.1101/gad.261982.115.

47. Sattiraju A, Sai K, Mintz A. Glioblastoma Stem Cells and Their Microenvironment. ADV EXP MED BIOL. 2017;1041:119-40. doi:10.1007/978-3-319-69194-7_7.

48. Bao B, Ahmad A, Li Y, Azmi AS, Ali S, Banerjee S, Kong D, Sarkar FH. Targeting CSCs within the tumor microenvironment for cancer therapy: a potential role of mesenchymal stem cells. Expert Opin Ther Targets. 2012;16:1041-54. doi:10.1517/14728222.2012.714774.

49. Cheng L, Huang Z, Zhou W, Wu Q, Donnola S, Liu JK, Fang X, Sloan AE, Mao Y, Lathia JD, Min W, McLendon RE, Rich JN, Bao S. Glioblastoma stem cells generate vascular pericytes to support vessel function and tumor growth. CELL. 2013;153:139-52. doi:10.1016/j.cell.2013.02.021.

50. Zhou W, Chen C, Shi Y, Wu Q, Gimple RC, Fang X, Huang Z, Zhai K, Ke SQ, Ping YF, Feng H, Rich JN, Yu JS, Bao S, Bian XW. Targeting Glioma Stem Cell-Derived Pericytes Disrupts the Blood-Tumor Barrier and Improves Chemotherapeutic Efficacy. CELL STEM CELL. 2017;21:591-603.e4. doi:10.1016/j.stem.2017.10.002.

51. Diaz RJ, Ali S, Qadir MG, De La Fuente MI, Ivan ME, Komotar RJ. The role of bevacizumab in the treatment of glioblastoma. J Neurooncol. 2017;133:455-67. doi:10.1007/s11060-017-2477-x.

\section{Figures}


A

D
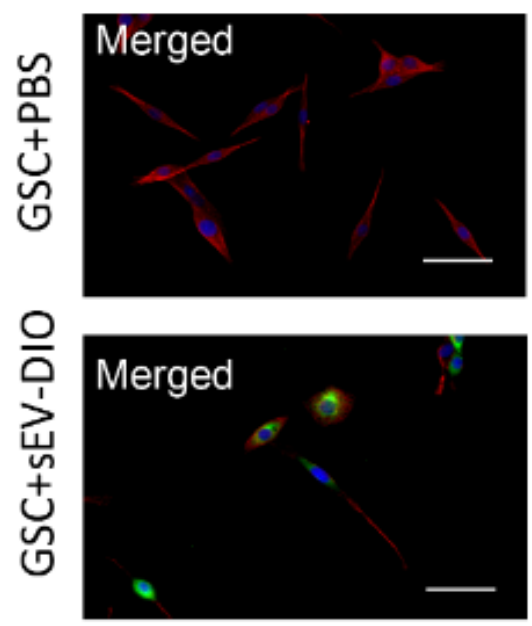

E
B

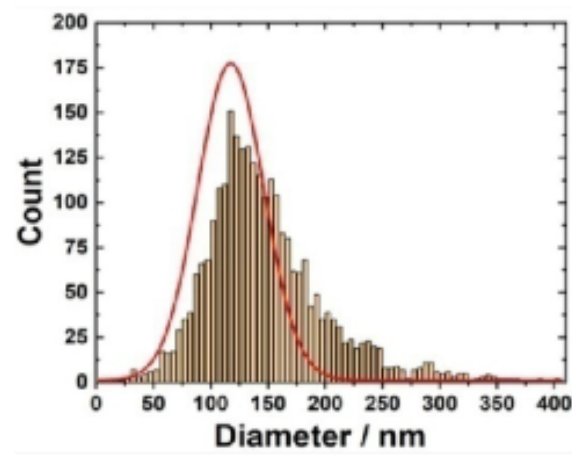

C
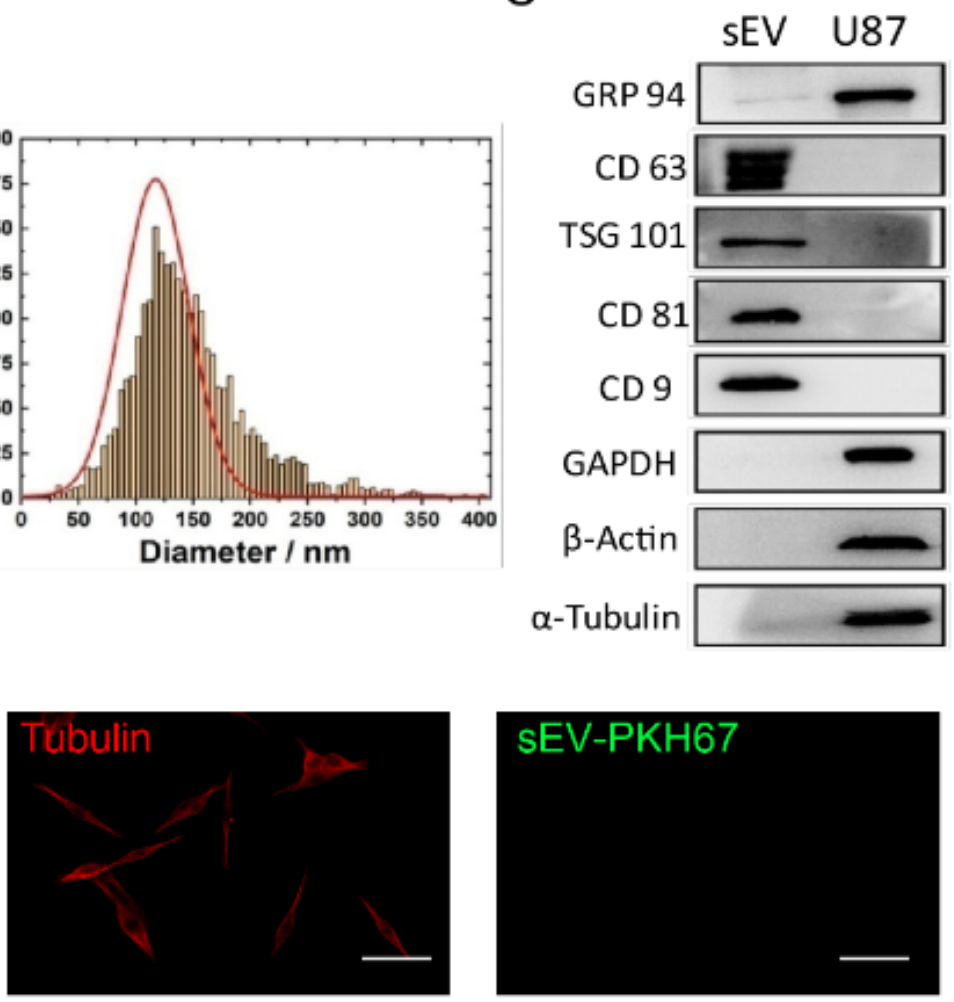

\section{SEV-PKH67}

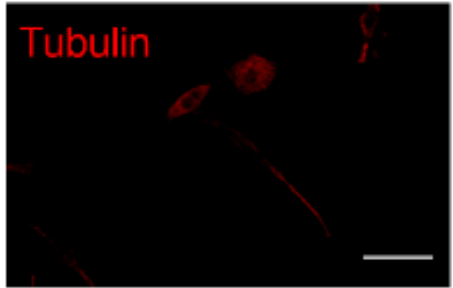

sEV-PKH67

$\sigma$



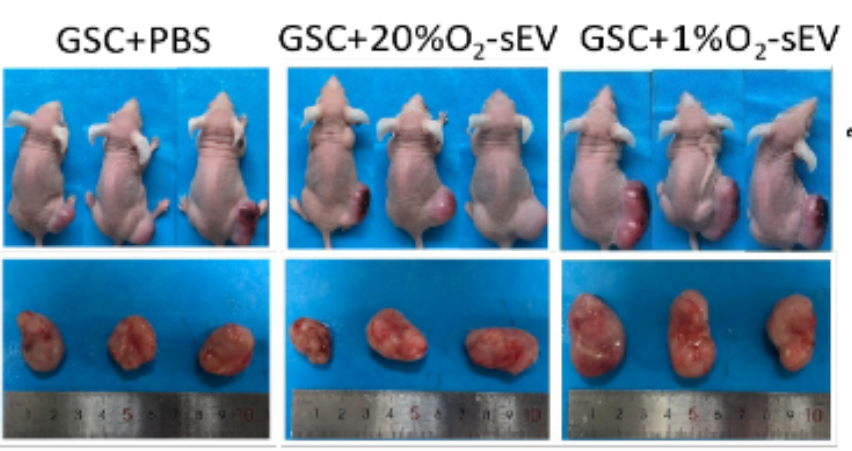

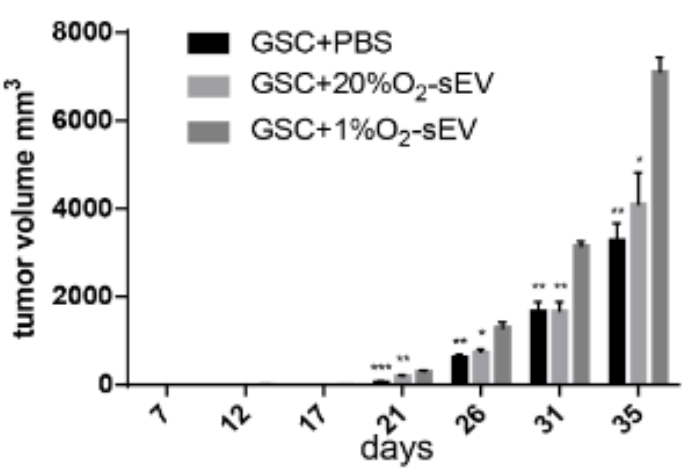

\section{Figure 1}

GSCs internalizes hypoxia-induced glioma-derived SEVs and promotes tumor growth in a mouse Xenograft. (A). Transmission electron microscopy (TEM) showing the morphology of sEV. (B).Size distribution of sEV attained from NanoSight particle tracking analysis (NTA). (C). Western blot was performed to evaluate the protein levels of sEV markers, including CD63, TSG101, CD81, and CD9, and negative marker, including GRP94, GAPDH, $\beta$-actin, and a-tubulin. (D). Immunofluorescence detected the 
lipophilic dye stained sEVs and corresponding GSCs. (scale bar $=50 \mathrm{um}$ ) (E). Representative images of U87 derived GSCs xenografts treated with PBS, normoxia and hypoxia-derived sEVs》respectively. The statistical chart of tumor volume was showed on the right. Data are means $\pm S D$. ${ }^{\star}, P<0.05 . * *, P<0.01$. $* \star *$, $\mathrm{P}<0.001$.
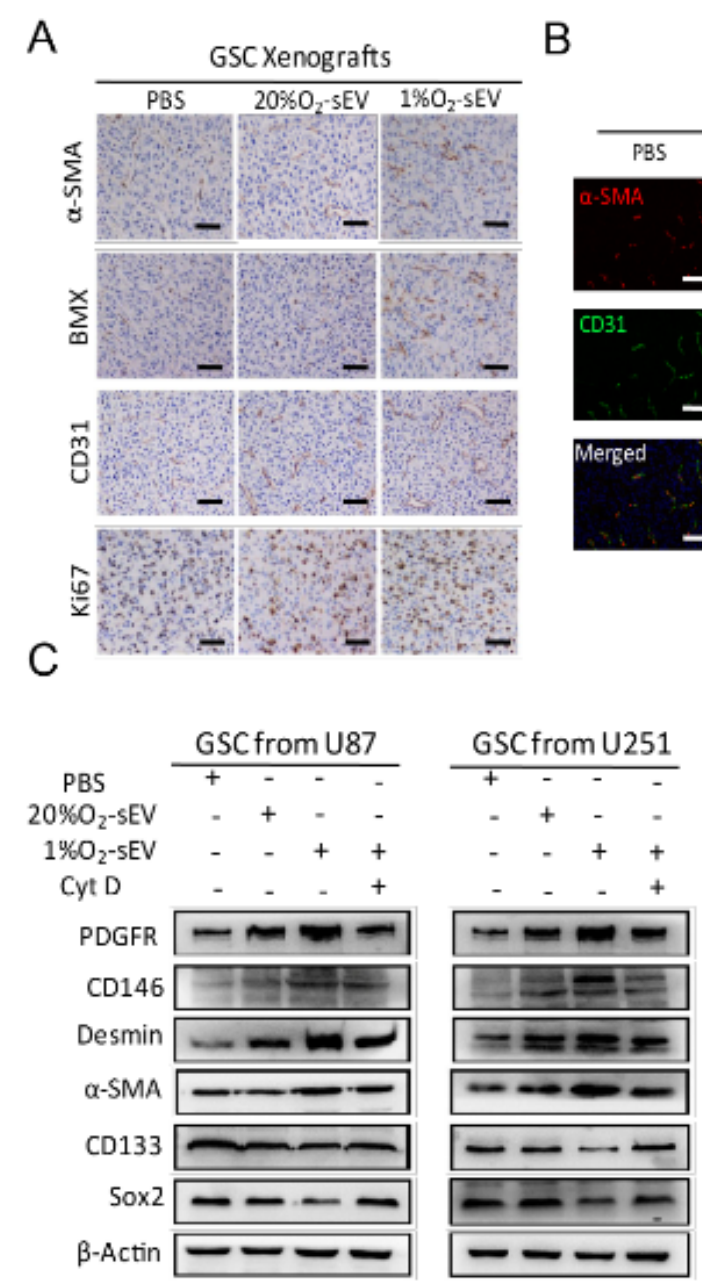

E
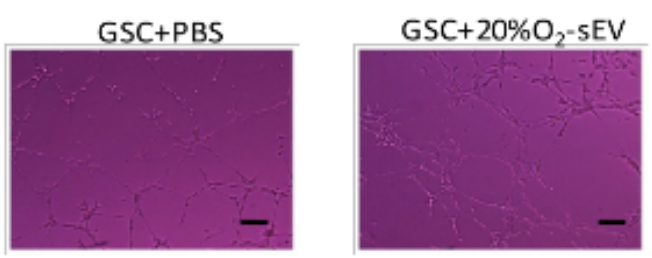

F

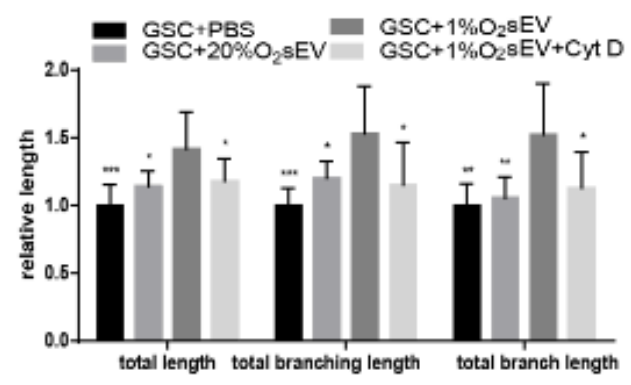

B
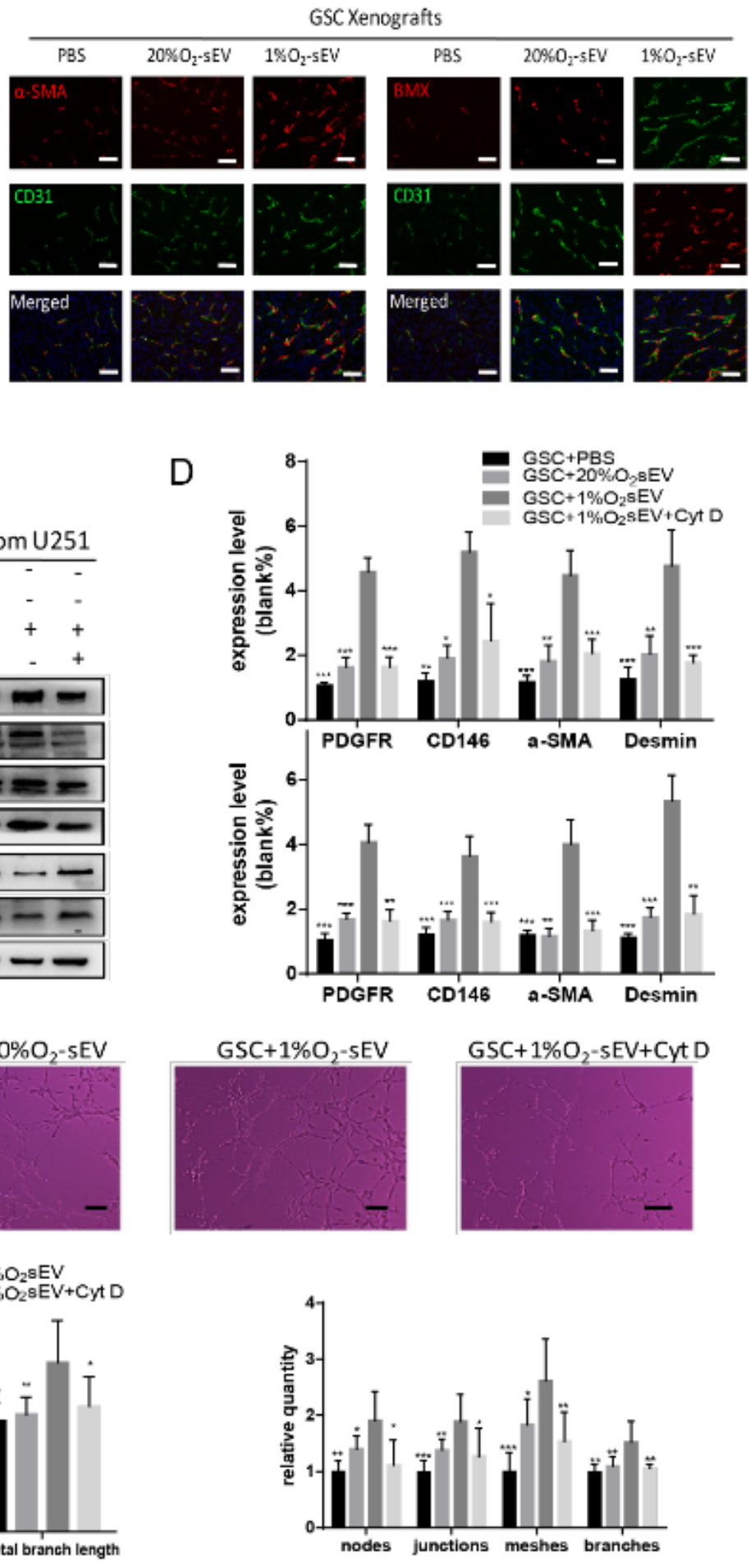

Figure 2 
The pericyte-phenotype transition of GSCs is induced by sEVs derived from hypoxic glioma cells. (A-B). Representative immunohistochemical and immunofluorescence images of CD31,a-SMA囚BMX and Ki67 in GSC xenografts, whcih were treated with PBS, normoxia and hypoxia-derived sEVs囚respectively. (scale bar $=100 \mathrm{um}$ in A; scale bar $=50$ um in B) (C). Western blot assay tested the expression of PDGFR, CD146, Desmin, a-SMA『CD133 and SOX2 in U87 /U251 derived GSCs treated with PBS, normoxia and

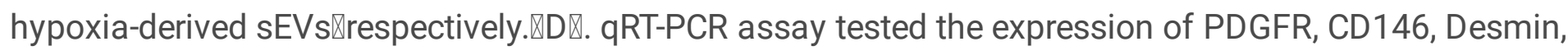
and a-SMA in U87 /U251 derived GSCs treated with PBS, normoxia and hypoxia-derived SEVs $\mathbb{Z}$ respectively. (E). Representative images of tubule formation ability in U87 derived GSCs treated with PBS, normoxia and hypoxia-derived sEVs $₫$ respectively. (scale bar $=100 \mathrm{um})(\mathrm{F})$. The statistical chart of tubule formation parameters, including junctions, mashes, nodes, total branching length, total segment length, and total length. Data are means $\pm S D .{ }^{*}, P<0.05$. ${ }^{\star}, P<0.01$. $* \star \star, P<0.001$. 
A
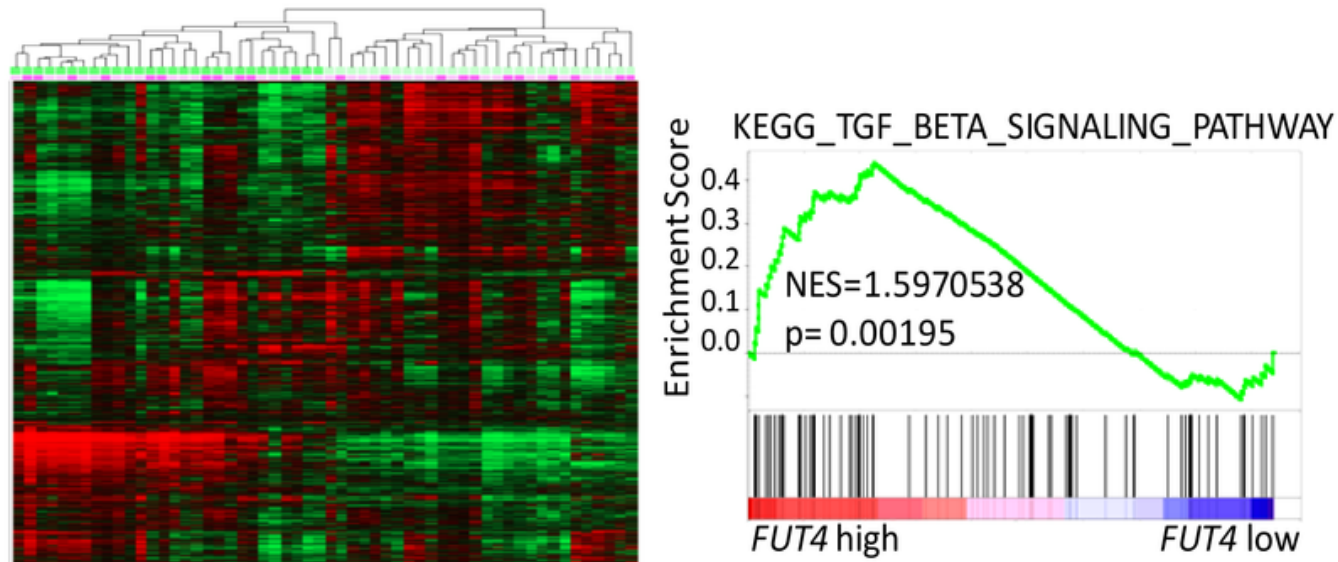

Expression level:

Absent

B

C
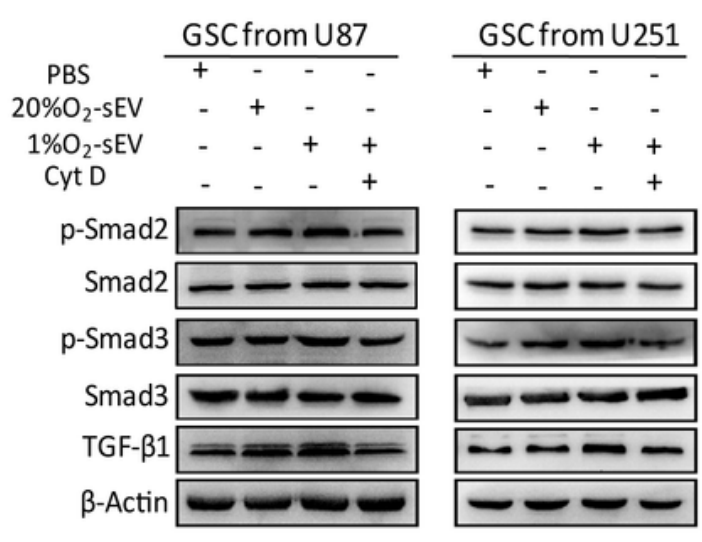

D
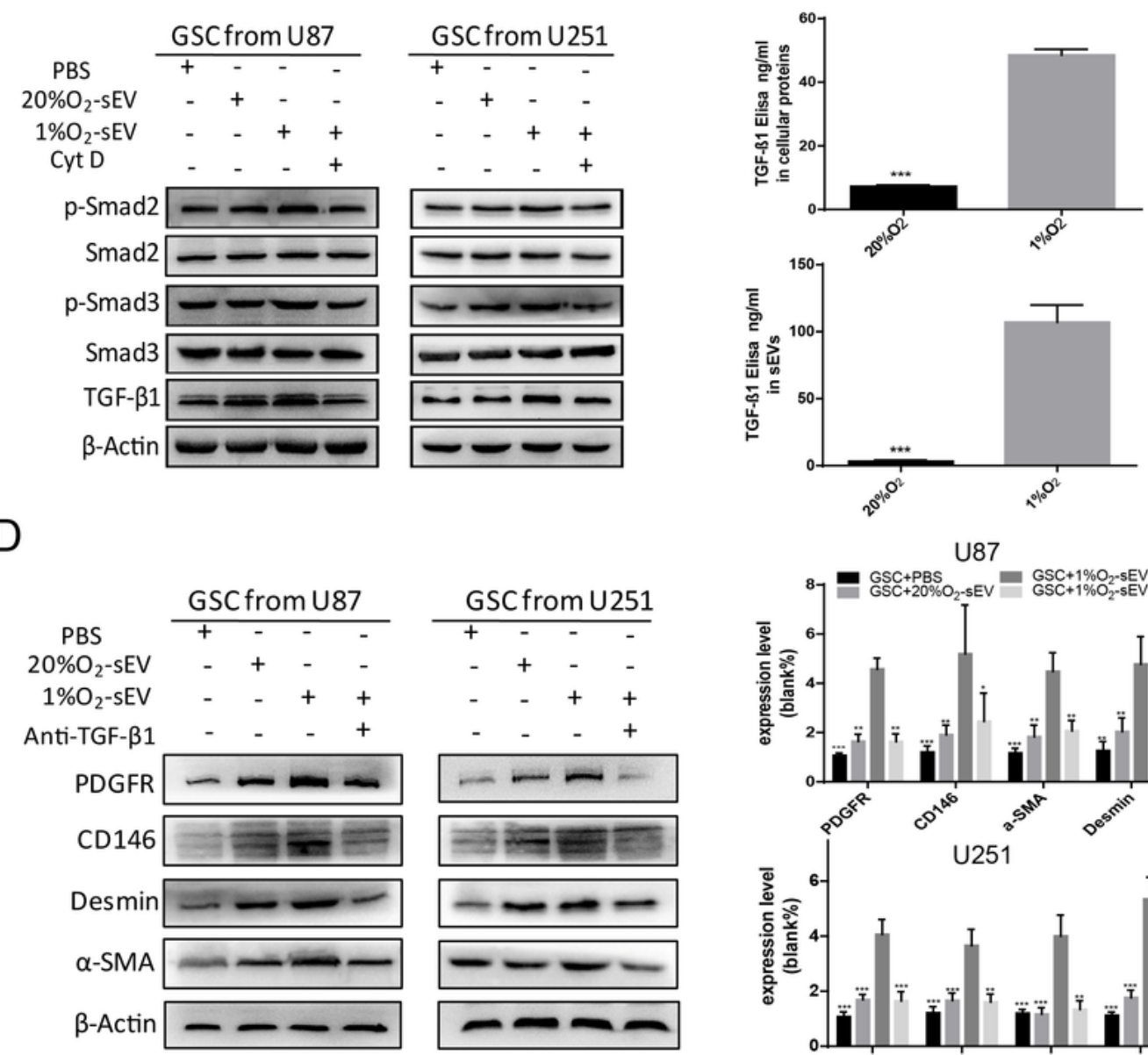

U87



Figure 3

TGF- $\beta 1$ signaling pathway mediates the pericyte-phenotype transition of GSCs. (A). A microarray containing intact mRNA of 56 polyclonal and monoclonal glioma stem cell lines downloaded from GEO Database (http:// www.ncbi.nlm.nih.gov/geo/query/acc.cgi?acc=GSE101113) was analyzed with GSEA enrichment plots of KEGG_TGF_BETA_SIGNALING_PATHWAY. (B) Western blot assay tested the expression of TGF- $\beta 1$, p-Smad2, p-Smad3, Smad2 and Smad3 in U87/U251 derived GSCs treated with 
PBS, normoxia and hypoxia-derived sEVs囚respectively. (C) Western blot assay tested the expression of PDGFR, CD146, Desmin, and a-SMA in U87/U251 derived GSCs treated with PBS, normoxia, hypoxiaderived sEVs『and combination of hypoxia-derived sEVs and Anti- TGF- $\beta 1$ respectively.

A

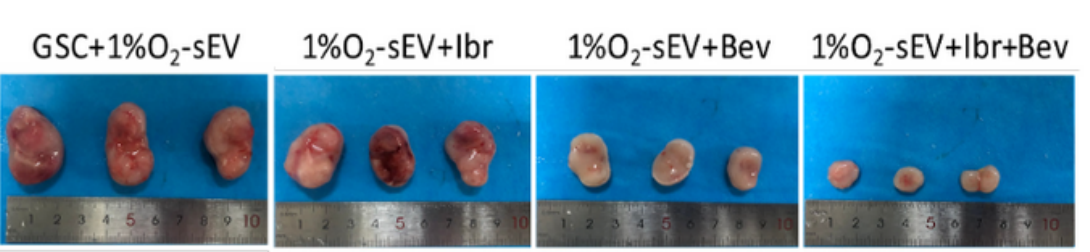

B

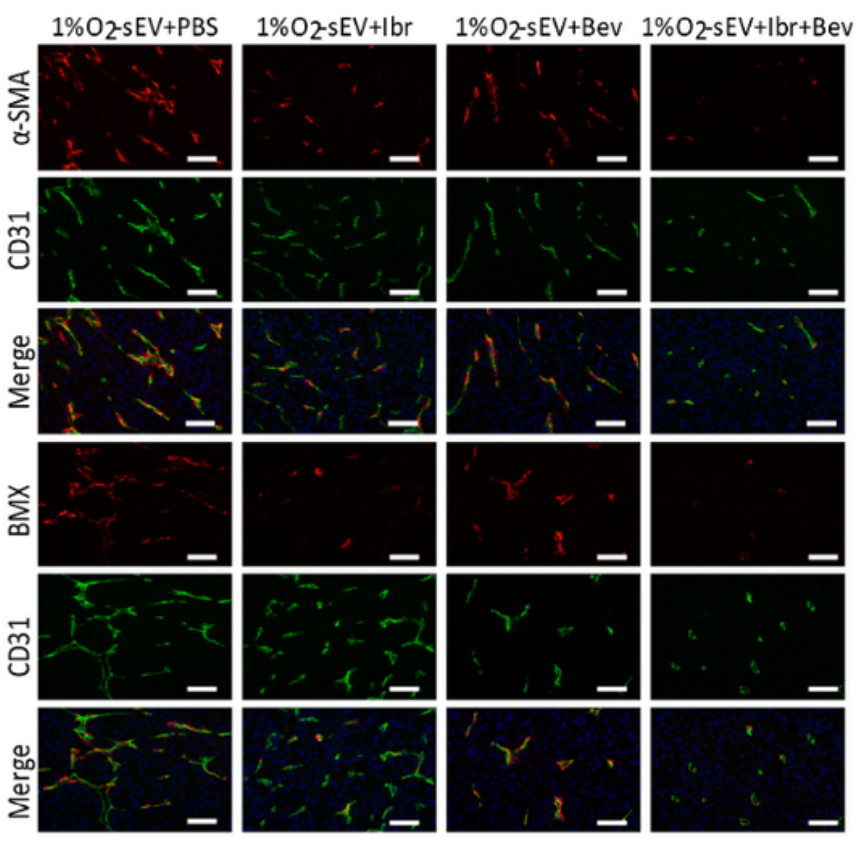

C

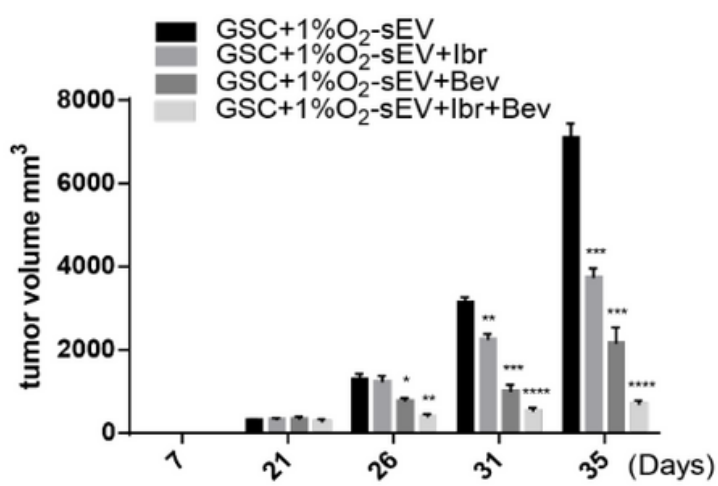

\section{Figure 4}

Ibrutinib, Bevacizumab and combined treatment of these two inhibit tumor growth in vivo. (A). Representative images of U87 derived GSCs xenografts treated with Ibrutinib, Bevacizumab and combined treatment of these two, respectively. The statistical chart of tumor volume was showed on the right. (B-C). Representative immunohistochemical and immunofluorescence images of CD31, a-SMA》 BMX and Ki67 in GSC xenografts, whcih were treated with Ibrutinib, Bevacizumab and combined treatment of these twoखrespectively. Data are means \pm SD. ${ }^{*}, P<0.05 . \star *, P<0.01$. ***, $P<0.001$.

\section{Supplementary Files}

This is a list of supplementary files associated with this preprint. Click to download.

- SupplymentaryFigure.docx

- SupplymentaryFig.1.png 